\title{
Résistivité électrique et effet Hall de métaux et semiconducteurs fondus
}

\section{Doctoral Thesis}

Author(s):

Tièche, Yvan

Publication date:

1963

Permanent link:

https://doi.org/10.3929/ethz-a-000088483

Rights / license:

In Copyright - Non-Commercial Use Permitted 
Prom. Nr. 3286

\title{
Résistivité électrique et effet Hall de métaux et semiconducteurs fondus
}

\author{
THÈSE \\ présentée à l'Ecole Polytechnique Fédérale, Zürich, \\ pour l'obtention $d u$ \\ grade de Docteur ès Sciences naturelles \\ par \\ YVAN TIECHE \\ physicien diplômé EPF \\ de Reconvilier (Suisse)
}

Rapporteur: Prof. Dr. G. Busch Corapporteur: Prof. Dr. J. Müller

Springer-Verlag

Berlin - Göttingen $\cdot$ Heidelberg

1963 
On donne la description d'un appareillage pour la mesure de l'effet Hall et de la résistivité électrique de métaux et semiconducteurs fondus. La méthode est basée sur la multiplication d'un champ magnétique et d'un courant alternatifs. Une cellule de mesure en silice avec cinq électrodes de graphite permet d'atteindre $1000^{\circ} \mathrm{C}$. Les résultats obtenus pour $\mathrm{Na}, \mathrm{Zn}, \mathrm{Cd}, \mathrm{Hg}$, $\mathrm{Ga}$, Ge et Sb sont explicables au moyen du modèle de l'électron libre. Les mobilités électroniques calculées correspondent aux valeurs mesurées. L'addition d'impuretés $(<1 \% \mathrm{Sb}$ ) dans le Te liquide n'a aucune influence sur ses propriétés électriques.

An apparatus is described for measuring the Hall effect and the electrical resistivity of molten metals and semiconductors, based on a cross-modulation method with alternating magnetic and electric fields. A quarz sample holder with five graphite electrodes may be used at temperatures up to $1000^{\circ} \mathrm{C}$. The results of experiments for $\mathrm{Na}, \mathrm{Zn}, \mathrm{Cd}, \mathrm{Hg}, \mathrm{Ga}$, Ge and $\mathrm{Sb}$ are consistent with the values given by the free-electron model. The calculated electronic mobilities are in agreement with measured values. The addition of small quantities of impurities $(<1 \% \mathrm{Sb})$ into molten Te does not affect its electrical properties.

Eine empfindliche Wechselfeld-Wechselstrom-Apparatur zur Messung des Hall-Effektes und des elektrischen Widerstandes von geschmolzenen Metallen und Halbleitern wird beschrieben. Der Probenhalter aus Quarz mit fünf Graphitsonden kann bis $1000^{\circ} \mathrm{C}$ benutzt werden. Die Messungen für $\mathrm{Na}, \mathrm{Zn}, \mathrm{Cd}, \mathrm{Hg}, \mathrm{Ga}$, Ge und Sb können erklärt werden mit dem Modell freier Elektronen. Die berechneten Werte der Elektronen-Beweglichkeiten stimmen mit den gemessenen überein. Die elektrischen Eigenschaften des flüssigen Te sind unabhängig von kleinen Verunreinigungskonzentrationen $(<1 \% \mathrm{Sb})$.

\section{A. Situation du problème}

Les énormes progrès réalisés dans l'étude du corps solide sont dus autant à la quantité et aux raffinements des recherches expérimentales qu'à l'élaboration de théories toujours plus évoluées. Contrastant avec ce développement très rapide, l'étude des substances fondues est restée à un stade assez peu avancé. Les propriétés physiques des métaux, semiconducteurs et électrolytes à l'état liquide sont encore mal connues et ne peuvent être expliquées que d'une façon très fragmentaire. Cette carence des connaissances de l'état liquide de la matière peut s'expliquer par les difficultés de l'application des techniques expérimentales usuelles aux matériaux fondus. Les températures élevées, les effets de corrosion, les mouvements hydrodynamiques ainsi que l'absence d'une géométrie définie font en effet surgir une quantité de problèmes nouveaux. Parallèlement, les théories reliant les propriétés physiques aux conceptions quantiques de la matière sont, jusqu'à ces derniers temps restées très limitées et pratiquement incontrôlables. 